\title{
Analysis of non-steroidal anti- inflammatory drugs in milk using QuEChERS and liquid chromatography coupled to mass spectrometry: triple quadrupole versus Q-Orbitrap mass analyzers
}

AntoniRúbies 1,*Emailarubies@aspb.cat

LiliGuo 1,2

FrancescCentrich 1,3

MercèGranados 2

1Laboratori de l'Agència de Salut Pública de BarcelonaAvinguda Drassanes 131508001BarcelonaSpain

2Department de Química AnalíticaUniversitat de BarcelonaMartí Franquès 11108028BarcelonaSpain

3CIBER of Epidemiology and Public Health (CIBERESP)28029MadridSpain Abstract

We developed a Quick, Easy, Cheap, Effective, Rugged, and Safe (QuEChERS) method for the high throughput determination of 10 non-steroidal antiinflammatory drugs (NSAIDs) in milk samples using high performance liquid chromatography-tandem mass spectrometry (HPLC-MS/MS) with a triple quadrupole $(\mathrm{QqQ})$ instrument and an electrospray ionization (ESI) source. The new extraction procedure is highly efficient, and we obtained absolute recoveries in the range $78.1-97.1 \%$ for the extraction and clean-up steps. Chromatographic separation is performed in the gradient mode with a biphenyl column and acidic mobile phases consisting of water and acetonitrile containing formic acid. The chromatographic run time was about $12 \mathrm{~min}$, and NSAID peaks showed a good symmetry factor. For MS/MS detection, we used multiple reaction monitoring (MRM) mode, using ESI in both positive and negative modes. Our method has been validated in compliance with the European Commission Decision 657/2002/EC, and we obtained very satisfactory results in inter-laboratory testing. Furthermore, we explored the use of a hybrid high resolution mass spectrometer, combining a quadrupole and an Orbitrap mass analyzer, for high resolution (HR) MS/MS detection of NSAIDs. We achieved lower NSAID quantification limits with QOrbitrap high resolution mass spectrometry (HRMS/MS) detection than those achieved with the QqQ instrument; however, its main feature is its very high selectivity, which makes HRMS/MS particularly suitable for confirmatory analysis. Keywords 
Liquid chromatography

Mass spectrometry

Triple quadrupole

Q-Orbitrap

Milk

Non-steroidal anti-inflammatory drugs

Electronic supplementary material

The online version of this article (doi: 10.1007/s00216-016-9679-5) contains supplementary material, which is available to authorized users.

\section{Introduction}

Non-steroidal anti-inflammatory drugs (NSAIDs) are widely used for their antiinflammatory, analgesic, and antipyretic properties. The most common side effects of NSAIDs are related to gastric and intestinal disturbances, but allergies and other effects involving the hepatic, renal, hematopoietic, or central nervous systems may also occur [1].

In the European Union (EU), some NSAIDs are authorized for administration to food-producing animals. Since residues of these compounds in edible products are a potential risk to consumers, the EU has established maximum residue limits (MRLs) for several NSAIDs in food products of animal origin [2]. Furthermore, the EU Community Reference Laboratories have proposed recommended concentrations (RC) for NSAID without MRL, and also for diclofenac [3]. These $\mathrm{RCs}$ are guidance values that laboratories need to achieve in order to ensure effective control. Table 1 shows MRL and RC values for NSAIDs in milk.

\section{Table 1}

Maximum residue limits (MRLs) and recommended concentrations (RCs) for NSAIDs

\begin{tabular}{|c|c|c|c|}
\hline \multirow{2}{*}{ Compound } & \multicolumn{2}{|c|}{ MRL in milk $\left(\mu \mathrm{g} \cdot \mathbf{k g}^{-1}\right)$} & \multirow{2}{*}{$\mathrm{RC}$ in mil } \\
\hline & Bovine & Caprine & \\
\hline 5-Hidroxyflunixin & 40 & & \\
\hline Diclofenac & 0.1 & & 5 \\
\hline Meloxicam & 15 & 15 & \\
\hline Phenylbutazone & & & 5 \\
\hline
\end{tabular}




\section{Table 1}

Maximum residue limits (MRLs) and recommended concentrations (RCs) for NSAIDs

MRL in milk $\left(\mu \mathrm{g} \cdot \mathbf{k g}^{-1}\right)$

Compound

Oxyphenbutazone

According to EU legislation, plans for controlling the presence of NSAID residues in food of animal origin are mandatory and, therefore, control laboratories require reliable, high throughput analytical methods. Few confirmatory methods have been developed for analyzing NSAID in milk samples. They are normally based on liquid chromatography (LC) coupled to mass spectrometry (MS), mostly using triple quadrupole (QqQ) instruments [4-9], although the use of high resolution mass spectrometry (HRMS, i.e. time of flight MS) and gas chromatography coupled to MS have also been described $[10,11]$.

NSAIDs are a heterogeneous group of drugs with multiple chemical structures, which can be classified into several groups (Table 2). This makes the development of NSAID multi-residue methods a challenging task, especially in terms of sample treatment (i.e., extraction and clean-up).

\section{Table 2}

Structure and molecule formulae of NSAIDs

Few solvents have been proposed for NSAID extraction. Acetonitrile, the effect of which on protein precipitation facilitates efficient extraction, is the most widely used solvent [4-6, 11]. However, methanol [7] as well as acetonitrile/methanol [12] or acetonitrile/ethyl acetate mixtures [9] are also employed, and good overall recovery values have been reported for all systems. A controversial issue concerning the extraction step is the addition of ascorbic acid to prevent oxidation of pyrazolones (e.g., phenylbutazone and oxyphenbutazone [11, 12]. Some authors report that it does not improve recovery [5] and may even have a detrimental effect on MS detection of other analytes [7]. The use of ascorbic acid at low concentration levels could solve this drawback [9].

Since LC-MS is susceptible to matrix effects, and milk is quite a complex matrix, most methods include some clean-up of the extracts. Several strategies have been described, such as a liquid-liquid extraction with hexane to eliminate fat content 
$[4,9]$, but most of the methods apply solid phase extraction (SPE) with different sorbents, depending on the set of compounds included in the method, such as amino [6], octadecyl [12], or polymeric phases [5]. In general, the clean-up step is the bottleneck of the procedure. The methods proposed by Dubreil-Chéneau et al. [7] and van Pamel and Daeseleire [8] are exceptions, and no clean-up was applied after extraction with methanol and acetonitrile, respectively.

LC-MS is clearly the technique of choice for confirmatory NSAID residue detection methods. Chromatographic separation is usually performed with octadecyl columns and mobile phases, mostly based on acetonitrile/water mixtures prepared at acidic $\mathrm{pH}$. Regarding MS detection, most of the methods use a QqQ instrument with an ESI source, in positive or negative mode, depending on the compound.

The overall aim of this study was to develop a reliable and straightforward methodology for the analysis of NSAID residues in milk that is suitable for routine use in laboratories with high workloads. Table 2 shows the chemical structure and molecular formula of the representative compounds from diverse NSAID families included in the present study. We present a new method based on the Quick, Easy, Cheap, Effective, Rugged and Safe (QuEChERS) strategy [13] and LC-QqQMS/MS to analyze 10 NSAIDs in milk. We also evaluated the detection by HRMS/MS with a Q-Orbitrap instrument.

\section{Materials and methods}

\section{Reagents}

All standards had over $98 \%$ purity. Standards of ketoprofen (KTP), niflumic acid (NFL), flufenamic acid (FLF), meclofenamic acid (MEC), flunixin (FLU), 5hydroxy-flunixin (5-FLU), oxyphenbutazone (OPB), phenylbutazone (PBZ), diclofenac (DCF), and meloxicam (MLX) were obtained from Sigma-Aldrich (Seelze, Germany). We used meloxicam- $\mathrm{D}_{3}\left(\mathrm{MLX}-\mathrm{D}_{3}\right)$, niflumic acid- ${ }^{13} \mathrm{C}_{6}(\mathrm{NFL}-$ ${ }^{13} \mathrm{C}_{6}$ ), flufenamic acid- ${ }^{13} \mathrm{C}_{6}\left(\mathrm{FLF}-{ }^{13} \mathrm{C}_{6}\right)$, phenylbutazone- $-{ }^{13} \mathrm{C}_{12}\left(\mathrm{PBZ}-{ }^{13} \mathrm{C}_{12}\right)$, also from Sigma-Aldrich, as internal standards (ISs). Acetonitrile and methanol (HPLC quality) were obtained from Panreac (Barcelona, Spain). Acetonitrile of hypergrade quality was obtained from Merck Millipore (Darmstadt, Germany). Double deionized water of $18.2 \mathrm{M} \Omega \cdot \mathrm{cm}^{-1}$ was obtained from a Milli-Q system (Millipore, Billerica, MA, USA). Other chemicals were of analytical reagent grade. Acetic acid and anhydrous sodium sulfate were obtained from Panreac. Ammonium acetate, ascorbic acid, and magnesium sulfate were obtained from Merck Millipore. Formic acid was obtained from Sigma-Aldrich (Seelze, Germany). Hydrochloric acid (32\%) was obtained from Fisher Chemical (Boston, MA, USA). Lichroprep RP-18 (25-40 $\mu \mathrm{m})$ was obtained from Merck Millipore, and Bondesil primary-secondary amines (PSA) 40-100 $\mu \mathrm{m}$ was obtained from Agilent Technologies, (New Castle, DE, USA); $0.22 \mu \mathrm{m}$ nylon membrane filters were obtained from Merck Millipore. 
Standard solutions were prepared as follows: individual stock solutions $\left(1000 \mathrm{mg} \cdot \mathrm{L}^{-1}\right): 10 \mathrm{mg}$ of each compound was weighed and dissolved in $10 \mathrm{~mL}$ of methanol using volumetric flasks, and stored at $-18{ }^{\circ} \mathrm{C}$ for up to $1 \mathrm{y}$. An intermediate standard solution at a concentration of $20 \mathrm{mg} \cdot \mathrm{L}^{-1}$ was prepared in methanol and stored for up to $6 \mathrm{mo}$ at $-18{ }^{\circ} \mathrm{C}$. Working solutions at concentrations of 5, 2, and $0.4 \mathrm{mg} \cdot \mathrm{L}^{-1}$ of each analyte were prepared in $0.1 \%$ formic acid in ultrapure water and stored for up to $3 \mathrm{mo}$ at $-18{ }^{\circ} \mathrm{C}$.

IS solutions were prepared in the same way as the stock standard solutions. The working solution had a concentration of $20 \mathrm{mg} \cdot \mathrm{L}^{-1}$.

Solutions of ascorbic acid $0.02 \mathrm{M}$, hydrochloric acid $0.24 \mathrm{M}$, and formic acid $0.1 \%$ were prepared using double deionized water as solvent.

\section{Instrumentation}

For sample preparation, we used a multi-tube vortex (VWR, DVX-2500), a laboratory centrifuge HettichRotanta 460R (Tuttlingen, Germany), a vertical agitator Agitax (Cisco Systems, Spain), a Turbovap nitrogen evaporator from Biotage (Uppsala, Sweden), and an ultrasonic bath (Ultrasons-H) from Selecta (Barcelona, Spain).

\section{LC-QqQ-MS/MS}

The LC system consisted of an Agilent Technologies 1290 coupled to an Agilent QqQ 6460 mass spectrometer with electrospray ionization (ESI), used in both positive and negative modes. The ESI source was operated under the following conditions: capillary voltage: $-3.0 \mathrm{kV}$ (ESI-) and $3.5 \mathrm{kV}$ (ESI+); sheath gas temperature: $375^{\circ} \mathrm{C}$; gas temperature: $180{ }^{\circ} \mathrm{C}$; gas flow $\left(\mathrm{N}_{2}\right): 5 \mathrm{~L} \cdot \mathrm{min}^{-1}$, sheath gas flow $\left(\mathrm{N}_{2}\right)$ : $11 \mathrm{~L} \cdot \mathrm{min}^{-1}$; nebulizer gas $\left(\mathrm{N}_{2}\right)$ pressure: 45 psi. Nitrogen was obtained from a Peek nitrogen generator (Air Liquid, Paris, France). Instrument control and data processing were carried out using Masshunter B.07.00 software.

A gradient using $0.1 \%$ formic acid in water (mobile phase A) and $0.1 \%$ formic acid in acetonitrile (mobile phase $B$ ) at a flow of $0.4 \mathrm{~mL} / \mathrm{min}$ was used to separate the NSAIDs on a Phenomenex Kinetex Biphenyl column $(2.6 \mu \mathrm{m}, 100 \times 2.1 \mathrm{~mm})$; the column temperature was kept at $40{ }^{\circ} \mathrm{C}$ and the injection volume was $20 \mu \mathrm{L}$. The gradient program was the following: (time, \%A): $(0,90),(10,45),(10.1,90),(14$, 90). The total runtime was $14 \mathrm{~min}$.

We monitored two transitions per compound and one transition for the internal standards. Table 3 shows the MS/MS parameters for each compound.

\section{Table 3}

Optimized MS parameters for QqQ and Q-Orbitrap 


\begin{tabular}{|c|c|c|c|c|c|c|c|c|c|}
\hline \multirow[b]{2}{*}{ Analyte } & \multicolumn{8}{|c|}{ QqQ } & \\
\hline & ESI+/ESI- & $\begin{array}{c}\mathbf{R t} \\
(\mathbf{m i n})\end{array}$ & $\begin{array}{l}\text { Precursor } \\
\text { ion }(m / z)\end{array}$ & $\begin{array}{c}\text { Fragmentor } \\
\operatorname{energy}(\mathbf{V})\end{array}$ & $\begin{array}{l}\text { Product } \\
\text { ion } 1 \\
(m / z)\end{array}$ & $\begin{array}{l}\mathbf{C E} \\
(\mathbf{V})\end{array}$ & $\begin{array}{c}\text { Product } \\
\text { ion } 2 \\
(m / z)\end{array}$ & $\begin{array}{l}\text { CE } \\
\text { (V) }\end{array}$ & HESI+/HES \\
\hline KTP & ESI+ & 7.11 & 254.8 & 130 & 105 & 15 & 77 & 50 & HESI+ \\
\hline NFL & ESI- & 7.39 & 280.6 & 130 & 176.8 & 15 & 237 & 35 & HESI- \\
\hline FLF & ESI- & 9.32 & 280.0 & 130 & 236.0 & 25 & 215.0 & 15 & HESI- \\
\hline MEC & ESI+ & 5.89 & 296.7 & 110 & 278.9 & 15 & 263.8 & 40 & HESI- \\
\hline FLU & ESI- & 5.89 & 294.5 & 70 & 251 & 15 & 208.9 & 35 & HESI- \\
\hline 5-FLU & ESI+ & 5.49 & 312.7 & 110 & 295 & 25 & 279.9 & 35 & HESI- \\
\hline OPB & ESI- & 7.29 & 323.0 & 130 & 295 & 15 & 134 & 15 & HESI- \\
\hline PBZ & ESI- & 9.47 & 307.0 & 130 & 279.1 & 15 & 131 & 15 & HESI- \\
\hline DCF & ESI- & 8.84 & 293.8 & 90 & 250.1 & 10 & 293.8 & -- & HESI- \\
\hline MLX & ESI+ & 7.51 & 351.6 & 130 & 115 & 15 & 141 & 25 & HESI+ \\
\hline $\begin{array}{l}\text { MLX- } \\
D_{3}\end{array}$ & ESI+ & 7.49 & 354.6 & 130 & 115 & 15 & --- & --- & HESI+ \\
\hline $\mathrm{NFL}^{13} \mathrm{C}_{6}$ & ESI- & 7.39 & 287.8 & 130 & 270 & 25 & --- & --- & HESI- \\
\hline $\mathrm{FLF}^{13} \mathrm{C}_{6}$ & ESI- & 9.324 & 286.1 & 130 & 242.1 & 15 & --- & --- & HESI- \\
\hline $\mathrm{PBZ}^{13} \mathrm{C}_{12}$ & ESI- & 9.53 & 320.8 & 130 & 166.1 & 25 & ---- & --- & HESI- \\
\hline
\end{tabular}

$C E$ collision energy, $N C E$ normalized collision energy, In bold quantitation product ion

\section{LC-Q-Orbitrap-HRMS/MS}

The HRMS instrument was a hybrid Q-Exactive from Thermo Scientific (Bremen, Germany). A Thermo Accela UHPLC system coupled to a Maylab Switch column manager and to the Q-Exactive mass spectrometer was used. The chromatographic system was coupled to the MS with a heated electrospray ionization source II (HESI II). HESI II conditions were: spray voltage $+3.5 \mathrm{kV}$ (positive ionization) or 
$-3 \mathrm{kV}$ (negative ionization); sheath gas flow rate $\left(\mathrm{N}_{2}\right), 35$ (arbitrary units); capillary temperature, $300{ }^{\circ} \mathrm{C}$; S-lens rf level, 50; heater temperature, $350{ }^{\circ} \mathrm{C}$. Nitrogen obtained from a nitrogen generator Zefiro (Clantecnologica, Seville, Spain) was employed as both the collision and damping gas.

Mass calibration for Orbitrap was performed daily to ensure a working mass accuracy lower than or equal to $5 \mathrm{ppm}$. Pierce LTQ Velos ESI Positive ion and Pierce LTQ Velos ESI Negative ion calibration solutions (Thermo Fisher Scientific, Rockford, IL, USA) were used to calibrate the mass spectrometer. The resolution was set at 70,000 ( $\mathrm{m} / \mathrm{z}, 200, \mathrm{FWHM})$ at a scan rate of $2 \mathrm{~Hz}$, and the automatic gain control (AGC) was set at $2 \mathrm{e}^{5}$ with a maximum injection time set at $100 \mathrm{~ms}$.

Xcalibur 2.2 and Trace Finder 3.2 (Thermo Fisher Scientific, San Jose, CA, USA) were used for LC-HRMS control and data processing, respectively.

A gradient using aqueous $0.1 \%$ formic acid (mobile phase $\mathrm{A}$ ) and acetonitrile hypergrade with $0.1 \%$ formic acid (mobile phase $\mathrm{B}$ ) at a flow of $0.3 \mathrm{~mL} \cdot \mathrm{min}^{-1}$ was used to separate the NSAIDs on a Phenomenex Kinetex XB C18 column $(1.7 \mu \mathrm{m}$, $100 \times 2.1 \mathrm{~mm})$; the column temperature was kept at $40{ }^{\circ} \mathrm{C}$ and the injection volume was $10 \mu \mathrm{L}$. The gradient program was as follows; (time, $\% \mathrm{~A}):(0,70),(10,45)$, $(10.1,70),(12,70)$. Total runtime was $12 \mathrm{~min}$.

Specific detection conditions when using HRMS are given in Table 3. Sample treatment

Five $\mathrm{g}$ of milk sample was weighed in a centrifuge tube and $100 \mu \mathrm{L}$ of a $20 \mathrm{mg} \cdot \mathrm{L}^{-1}$ solution of IS was added. Subsequently, $10 \mathrm{~mL}$ of $5 \%$ acetic acid in $\mathrm{ACN}, 1 \mathrm{~g}$ of ammonium acetate, $4 \mathrm{~mL}$ of ascorbic acid $0.02 \mathrm{M}$ in $\mathrm{HCl} 0.24 \mathrm{M}$ and $5 \mathrm{~g}$ of $\mathrm{Na}_{2} \mathrm{SO}_{4}$ were added. The sample was shaken for $5 \mathrm{~min}$ using multi-tube vertical shaker, sonicated for $5 \mathrm{~min}$ and subsequently centrifuged $\left(3000 \mathrm{rpm}, 10^{\circ} \mathrm{C}\right.$, $10 \mathrm{~min}$ ). The supernatant was transferred to a centrifuge tube containing $150 \mathrm{mg}$ of $\mathrm{C} 18$ and $1 \mathrm{~g}$ of $\mathrm{MgSO}_{4}$ and the mixture was shaken for $5 \mathrm{~min}$ (vortex), sonicated for $5 \mathrm{~min}$, and centrifuged under the aforementioned conditions. Then, the supernatant was evaporated to around $250 \mu \mathrm{L}$ under a $\mathrm{N}_{2}$ stream at $40{ }^{\circ} \mathrm{C}$, diluted to $500 \mu \mathrm{L}$ with $0.1 \%$ formic acid and filtered through a $0.22 \mu \mathrm{m}$ nylon filter membrane. Calibration

The quantification of NSAIDs in milk samples was based on the use of surrogate matrix matched standards (SMMS). SMMSs consisted of blank milk samples spiked with known amounts of NSAIDs and $100 \mu \mathrm{L}$ of IS. After spiking, SMMSs were left for contact during $30 \mathrm{~min}$ before proceeding to the extraction, according to the extraction protocol described above. Linear regression was performed by plotting the peak area ratio of the analyte to IS against the analyte concentration. ISs were assigned to analytes as follows: MLX-D $\mathrm{D}_{3}$ for MLX, DCF, and KTP, NFL- ${ }^{13} \mathrm{C}_{6}$ for NFL and MEC, FLF-13C $C_{6}$ for FLF, 5-FLU, FLU, and PBZ- ${ }^{13} \mathrm{C}_{12}$ for PBZ and OPB. Calibration with SMMS provides quantification of the sample automatically corrected by the recovery value. 


\section{Results and discussion}

\section{Method development}

\section{LC-QqQ-MS/MS}

The multiple reaction monitoring (MRM) mode was used for quantification and confirmation. Thus, to optimize MS detection and select precursor and product ions for each analyte, standard solutions of $5 \mathrm{mg} \cdot \mathrm{L}^{-1}$ in methanol were infused into the mass spectrometer, and spectra were acquired using the ESI source operating in positive or negative mode. The mobile phase consisted of water:acetonitrile (1:1). The "Fragmentor" parameter was optimized in the 70-130 V range to obtain maximum response for precursor ions. Collision energy was optimized in the range 5-35 V to obtain maximum response for the product ions. The final selection of ESI polarity and MRM transitions for each NSAID was based on both sensitivity and selectivity criteria. The mass spectrometer was quick enough to switch polarity without compromising sensitivity. Two transitions of the precursor ion were selected to achieve enough identification points to confirm the identity of the analytes (Table 3). The most intense transition (in bold) was used for quantification, and the ion ratio parameter for confirmation.

Although MS detection is a selective technique, good chromatographic separation is advisable. The chromatographic separation of NSAIDs is usually performed in reversed mode on $\mathrm{C} 8$ or $\mathrm{C} 18$ columns. In this study, we assayed two chromatographic columns (Kinetex XB C18, $1.7 \mu \mathrm{m}$ and Kinetex biphenyl, $2.1 \mu \mathrm{m}$, both solid core type). The biphenyl stationary phase behaves similarly to C18 but with enhanced aromatic selectivity, so that deeper interaction with the aromatic rings of the analytes is achieved. We used mobile phases based on wateracetonitrile mixtures and tested several gradient programs at $0.4 \mathrm{~mL} \cdot \mathrm{min}^{-1}$, starting at $10 \%$ to $40 \%$ acetonitrile and reaching $55 \%$ acetonitrile in 6 to $10 \mathrm{~min}$. We observed some changes in the elution order between the columns: C18 column, MLX, 5-FLU, KTP, MEC, FLU, FLF, NFL, OPB, DCF, and PBZ; biphenyl column, 5-FLU, MEC, FLU, KTP, OPB, NFL, MLX, DCF, FLF, and PBZ. Some overlapping peaks were obtained with both columns, but the NSAID peaks obtained with the biphenyl column showed a better symmetry factor than those obtained with the $\mathrm{C} 18$ column and, thus, the biphenyl column was selected for further assays. When injecting milk extracts, NSAID retention times showed poor reproducibility when comparing injections of standards in solvent and standards in extracted matrix. Adding formic acid to the mobile phase resolved this issue, leading to reproducible retention times. The addition of acidic media did not result in loss of sensitivity for the compounds monitored in negative mode. After adjusting gradient conditions for the biphenyl phase (described in the Materials and methods Section), we achieved good chromatographic separation in a total run time of $14 \mathrm{~min}$, including equilibration time (Fig. 1). 


\section{Fig. 1}

LC-QqQ-MS/MS chromatograms of a standard mixture of $0.4 \mathrm{mg} \cdot \mathrm{L}^{-1}$ of NSAIDs and internal standards

\section{Extraction method}

Various approaches have been proposed for extracting NSAIDs from milk samples. Acetonitrile has been found to provide efficient extraction and quite clean extracts because of its deproteinizing effect. Methanol is less commonly used, but is also effective. Regardless, most methods include a clean-up step, based on either solid phase extraction or liquid-liquid extraction with hexane, or a combination of both, before the chromatographic analysis. The aim of this study was to develop a simple but reliable extraction method, suitable for laboratories with high workloads. Thus, we carried out preliminary studies using the QuEChERS approach, and also applied the methods proposed by Jedziniak et al. [6] (extraction with acetonitrile containing ammonia and clean-up by SPE with amino cartridge), and by Moragues et al. [14] (extraction with methanol and clean-up by SPE with C18 cartridge). In the QuEChERS assays, the extraction step was based on acetonitrile containing $5 \%$ acetic acid to ensure protonation of carboxylic acids. We also added sodium chloride and magnesium sulfate. After shaking and centrifugation, the organic layer underwent a clean-up step by dispersive solid phase extraction (d-SPE) with C18. We analyzed the extracts by LC-QqQ-MS/MS, and compared NSAID peak areas using the three methodologies. Overall, none of the methods assayed provided optimal results for all compounds, but yielded similar results when compared globally. Thus, the simplicity and speed of the QuEChERS methodology was a significant differentiating factor, so we focused our efforts on optimizing a new method based on this approach.

We compared two distinct dispersive media for the d-SPE step, C18 and PSA, and found better recoveries for the majority of analytes when using C18 (see Electronic Supplementary Material (ESM) Fig. S1). We also investigated to omit the clean-up step in order to speed up the analysis, but we obtained unsatisfactory results, with low signals for almost all analytes (see ESM Fig. S1)

Although the results achieved using the QuEChERS approach were satisfactory overall, MS signals for PBZ and OPB were low and poorly reproducible, both with $\mathrm{C} 18$ and PSA. These compounds may readily undergo oxidation, and the addition of ascorbic acid to prevent their oxidation has been proposed. However, this antioxidant reagent may have deleterious effects on MS/MS detection of NSAIDs [7], which can be prevented by using a relatively low concentration of ascorbic acid [9]. Adding $0.006 \mathrm{M}$ ascorbic acid to the acetonitrile extraction solution markedly improved the results of PBZ and OPB, and with no relevant adverse effects on the signals of other NSAIDs.

Finally, we substituted sodium chloride with ammonium acetate in the extraction solution, and obtained lower baseline noises, thus allowing lower detection limits. 
The final conditions adopted for the QuEChERS extraction and clean-up by d-SPE with $\mathrm{C} 18$ are reported in the Sample treatment Section. The absolute recoveries for the QuEChERS method obtained at $2.5 \mu \mathrm{g} \cdot \mathrm{kg}^{-1}$ are shown in Fig. S2 (ESM). These results, in the $78-96 \%$ range, are very satisfactory, making this a suitable and simple new method for routine laboratory testing.

\section{Method validation}

We validated the QuEChERS LC-QqQ-MS/MS method in milk according to the European Commission Decision 657/2002/EC guidelines [15], and tested the following parameters: linearity, extraction recovery, intermediate precision, trueness, selectivity, ruggedness, limit of decision $(\mathrm{CC} \alpha)$, and detection capability $(\mathrm{CC} \beta)$.

Linearity

We built calibration curves for each compound using SMMS in the 2.5$25 \mu \mathrm{g} \cdot \mathrm{kg}^{-1}$ range, except for FLU, for which calibration curves reached $60 \mu \mathrm{g} \cdot \mathrm{kg}^{-1}$. We used the correlation coefficient and point-to-point deviation (in \%, with respect to the theoretical value) to evaluate linearity, and obtained good linearity for all analytes: correlation coefficients $>0.99$ for all the compounds except for ketoprofen, which showed correlation coefficient $>0.95$. Residuals were always $<25 \%$ at the lowest concentration $\left(2.5 \mu \mathrm{g} \cdot \mathrm{kg}^{-1}\right)$ and $<15 \%$ for higher calibration concentrations.

Extraction recovery

Before extraction, we spiked a set of blank samples in the range 2.5$40 \mu \mathrm{g} \cdot \mathrm{kg}^{-1}$ and extracted. Then, we extracted and spiked a second set of blank samples after extraction and clean-up, and calculated absolute recovery for sample treatment steps by comparing the results. The experiment was repeated three times on three different days. Absolute recoveries for the extraction and clean-up steps ranged from 78.1 to $97.1 \%$ (Table 4).

\section{Table 4}

Validation results in milk

\begin{tabular}{|c|c|c|c|c|}
\hline Analyte & Concentration $\left(\mu \mathrm{g} \cdot \mathrm{kg}^{-1}\right)$ & $\begin{array}{l}\text { Extraction recovery }(\%) \\
\qquad(n=3)\end{array}$ & $\begin{array}{l}\text { Precision (RSD\%) } \\
\qquad(n=18)\end{array}$ & $\begin{array}{l}\text { Trueness } \\
\qquad(n=18\end{array}$ \\
\hline \multirow{2}{*}{ KTP } & 2.5 & 81.0 & 10.4 & 87.6 \\
\hline & 5.0 & 95.6 & 12.0 & 97.8 \\
\hline
\end{tabular}




\section{Table 4}

Validation results in milk

\begin{tabular}{|c|c|c|c|c|}
\hline Analyte & Concentration $\left(\mu \mathrm{g} \cdot \mathrm{kg}^{-1}\right)$ & $\begin{array}{l}\text { Extraction recovery }(\%) \\
\qquad(n=3)\end{array}$ & $\begin{array}{l}\text { Precision (RSD\%) } \\
\qquad(n=18)\end{array}$ & $\begin{array}{l}\text { Trueness } \\
\qquad(n=18\end{array}$ \\
\hline & 10.0 & 92.2 & 10.7 & 98.8 \\
\hline & 25.0 & 87.5 & 8.6 & 103.1 \\
\hline \multirow{4}{*}{ NFL } & 2.5 & 93.4 & 9.2 & 108.4 \\
\hline & 5.0 & 90.6 & 7.8 & 107.4 \\
\hline & 10.0 & 93.4 & 10.1 & 94.0 \\
\hline & 25.0 & 93.9 & 10.1 & 100.8 \\
\hline \multirow{4}{*}{ FLF } & 2.5 & 96.0 & 10.3 & 108.8 \\
\hline & 5.0 & 90.9 & 7.9 & 108.0 \\
\hline & 10.0 & 96.5 & 8.3 & 95.5 \\
\hline & 25.0 & 91.9 & 9.4 & 101.2 \\
\hline \multirow{4}{*}{ MEC } & 2.5 & 91.2 & 10.8 & 105.6 \\
\hline & 5.0 & 90.4 & 10.4 & 102.8 \\
\hline & 10.0 & 93.3 & 10.4 & 84.1 \\
\hline & 25.0 & 95.3 & 12.2 & 94.1 \\
\hline \multirow{4}{*}{ FLU } & 2.5 & 92.9 & 10.3 & 97.6 \\
\hline & 5.0 & 90.2 & 13.5 & 94.6 \\
\hline & 10.0 & 95.2 & 7.9 & 105.1 \\
\hline & 25.0 & 89.3 & 10.8 & 102.3 \\
\hline
\end{tabular}




\section{Table 4}

Validation results in milk

\begin{tabular}{|c|c|c|c|c|}
\hline Analyte & Concentration $\left(\mu \mathrm{g} \cdot \mathrm{kg}^{-1}\right)$ & $\begin{array}{l}\text { Extraction recovery (\%) } \\
\qquad(n=3)\end{array}$ & $\begin{array}{l}\text { Precision (RSD\%) } \\
\qquad(n=18)\end{array}$ & $\begin{array}{l}\text { Trueness } \\
\qquad(n=18\end{array}$ \\
\hline \multirow{5}{*}{ 5-FLU } & 2.5 & 89.7 & 14.3 & 91.2 \\
\hline & 5.0 & 87.1 & 9.7 & 103.8 \\
\hline & 10.0 & 88.2 & 11.5 & 85.7 \\
\hline & 25.0 & 88.2 & 10.0 & 101.1 \\
\hline & 40.0 & 88.3 & 1.7 & 101.9 \\
\hline \multirow{4}{*}{ DCF } & 2.5 & 92.3 & 9.2 & 92.4 \\
\hline & 5.0 & 91.0 & 8.8 & 100.0 \\
\hline & 10.0 & 97.1 & 12.8 & 102.0 \\
\hline & 25.0 & 90.9 & 12.2 & 90.8 \\
\hline \multirow{4}{*}{ DCF } & 2.5 & 92.3 & 9.2 & 92.4 \\
\hline & 5.0 & 91.0 & 8.8 & 100.0 \\
\hline & 10.0 & 97.1 & 12.8 & 102.0 \\
\hline & 25.0 & 90.9 & 12.2 & 90.8 \\
\hline \multirow{4}{*}{$\mathrm{OPB}$} & 2.5 & 94.7 & 11.7 & 103.2 \\
\hline & 5.0 & 89.3 & 13.1 & 94.6 \\
\hline & 10.0 & 91.1 & 12.2 & 98.8 \\
\hline & 25.0 & 85.6 & 13.7 & 100.7 \\
\hline PBZ & 2.5 & 78.1 & 16.9 & 98.8 \\
\hline
\end{tabular}


Table 4

Validation results in milk

\begin{tabular}{|c|c|c|c|c|}
\hline Analyte & Concentration $\left(\mu \mathrm{g} \cdot \mathbf{k g}^{-1}\right)$ & $\begin{array}{l}\text { Extraction recovery }(\%) \\
\qquad(n=3)\end{array}$ & $\begin{array}{l}\text { Precision (RSD\%) } \\
\qquad(n=18)\end{array}$ & $\begin{array}{l}\text { Trueness } \\
\qquad(n=18\end{array}$ \\
\hline & 5.0 & 89.1 & 12.6 & 101.8 \\
\hline & 10.0 & 95.3 & 11.8 & 99.1 \\
\hline & 25.0 & 86.1 & 14.9 & 88.9 \\
\hline \multirow{5}{*}{ MLX } & 2.5 & 94.4 & 15.2 & 98.0 \\
\hline & 5.0 & 92.5 & 9.0 & 102.8 \\
\hline & 10.0 & 92.0 & 7.8 & 102.0 \\
\hline & 15.0 & 91.2 & 3.0 & 103.1 \\
\hline & 25.0 & 90.5 & 12.2 & 104.3 \\
\hline
\end{tabular}

Intermediate precision

The study was performed at four concentration levels over $3 \mathrm{~d}$, with six spiked samples analyzed daily at each concentration level. Results were evaluated in terms of relative standard deviation (\%RSD), which ranged from 1.7 to16.9 \%, and were always below the value calculated by the Horwitz equation.

Trueness

There is no certified material available for determining trueness, so we used the spiked samples approach. We compared the concentrations obtained for the spiked blank samples with the theoretical values from a SMMS calibration curve, expressed in \%, and obtained good results, ranging from 84.1 to $108.8 \%$ (Table 4). Selectivity

We evaluated this parameter by analyzing different blank samples, and in all cases we observed no interferences at the retention time of the analytes. In addition, we found no relevant differences between the IS responses in all blank samples, with $\%$ RSD below $20 \%$. Ruggedness 
We evaluated critical steps of the method by introducing minor changes and evaluating the results. The parameters evaluated were the use of different samples, the effect of drying on the extracts, and the effect of the evaporation temperature. Moreover, the use of different instruments (vertical agitators and evaporators) was considered. Different operators carried out the experiments. No significant differences were found for the studied parameters, except for the evaporation step: when reaching dryness of the extracts, we obtained low responses for KTP, OPB, and PBZ.

$\mathrm{CC} \alpha$ and $\operatorname{CC} \beta$

MRL has only been established in milk for three of the studied compounds, namely FLU (expressed as 5-FLU), MLX, and DCF. For MLX and 5-FLU, CC $\alpha$ and CC $\beta$ were determined around these limits as described in [15], and results are shown in Table 4. However, for DCF, the MRL has been set at such a low concentration $\left(0.1 \mu \mathrm{g} \cdot \mathrm{kg}^{-1}\right)$ that the method was not suitable. In fact, very few methods have reported in the literature as being able to confirm the presence of DCF at $0.1 \mu \mathrm{g} \cdot \mathrm{kg}^{-1}[6,8,9]$.

We proceeded with DCF as for NSAIDs with no published MRL, with CC $\alpha$ and $\mathrm{CC} \beta$ were established at the lowest possible concentration level. Thus, we determined $\mathrm{CC} \alpha$ and $\mathrm{CC} \beta$ by extrapolating the calibration curve as described in 2002/657/EC Commission Decision [15], based on the ISO/IEC 11843-2 standard regulation [16]. In the present study, the SMMS calibration curves ranged from 2.5 to $25 \mu \mathrm{g} \cdot \mathrm{kg}^{-1}$. Results for $\mathrm{CC} \alpha$ and $\mathrm{CC} \beta$ ranged from 0.4 to $1.5 \mu \mathrm{g} \cdot \mathrm{kg}^{-1}$ for $\mathrm{CC} \alpha$ and from 0.8 to $1.9 \mu \mathrm{g} \cdot \mathrm{kg}^{-1}$ for $\mathrm{CC} \beta$ (Table 4).

To assess the performance of the method and the consistency of the calculated CC $\alpha$, blank milk samples were spiked at $0.5,1$, and $1.5 \mu \mathrm{g} \cdot \mathrm{kg}^{-1}$ and analyzed using the new method. The NSAIDs were detected in all cases.

Furthermore, we participated in the inter-laboratory exercise Fapas number 02274 (NSAIDs in bovine milk), with very satisfactory results: the sample contained $7 \mu \mathrm{g} \cdot \mathrm{kg}^{-1}$ of PBZ and a Z-score $=0$ was obtained, proving the suitability of the proposed method.

\section{Analysis of NSAIDs in milk by UHPLC-Q-Orbitrap-HRMS/MS}

The new generation of HRMS instruments, such as Orbitrap-based mass spectrometers, are a powerful tool for enhancing the selectivity of analytical methods because of their high resolution power and sensitivity. In the food safety field, approaches using these types of instruments are increasingly being used to confirm the results of low resolution mass spectrometers (LRMS), and also as a tool for routine analysis. They seem especially suited for highly demanding scenarios, such as the analysis of DCF in milk, because of its very low MRL $\left(0.1 \mu \mathrm{g} \cdot \mathrm{kg}^{-1}\right)$. We used a hybrid Q-Orbitrap instrument coupled to a UHPLC system. The chromatographic separation of NSAIDs was performed in the gradient elution mode on a Kinetex XB C18 column as described in the Materials and methods Section. 
Total run time was $15 \mathrm{~min}$ and retention times for NSAIDs ranged between 5.6 and 11 min.

Standards of $1 \mathrm{mg} \cdot \mathrm{L}^{-1}$ were injected into the UHPLC-Q-Orbitrap system to optimize the analytical response, for both precursor and product ions of NSAIDs. The working mode was product reaction monitoring (PRM) with a mass width for the precursor ion selection set at $1 \mathrm{Da}$ and resolution set at 70,000 ( $\mathrm{m} / \mathrm{z}, 200$, FWHM). We selected mild conditions for collision energy, allowing both precursor and product ion monitoring, and operational parameters were optimized (shown in theMaterials and methods Section). Comparing QqQ and Q-Orbitrap spectrometer working conditions, some compounds differ in polarity (i.e., MEC and 5-FLU). The majority of NSAIDs ionize in both positive and negative mode, and our criterion was to select conditions that provided the optimal response for each instrument to achieve the lowest concentrations.

Following chromatographic and MS optimization, we injected extracted milk samples to test the performance of the method. We analyzed SMMSs in the concentration range $2.5-40 \mu \mathrm{g} \cdot \mathrm{kg}^{-1}$ and obtained good linearity, with correlation factors higher than 0.99. All NSAIDs were detected and confirmed at the lowest calibration level.

Finally, we tested the performance of the UHPLC-HRMS/MS system at sub$\mu \mathrm{g} \cdot \mathrm{kg}^{-1}$ level. For this purpose, blank milk samples were spiked with NSAIDs at 1 , $0.7,0.5,0.3,0.1$, and $0.05 \mu \mathrm{g} \cdot \mathrm{kg}^{-1}$. All NSAIDs were detected at 1 and $0.7 \mu \mathrm{g} \cdot \mathrm{kg}^{-1}$ (both precursor and product ions were present); precursor ions were detectable at lower concentrations, even at $0.1 \mu \mathrm{g} \cdot \mathrm{kg}^{-1}$, except for MEC. However, confirmation at this level was only possible for KTP, NFL, FLF, FLU, 5-FLU, OPB, DCF, and MLX (Fig. 2). These results show that confirmatory analysis of DCF is possible at the MRL level. The mass accuracy was below $5 \mathrm{ppm}$ in all cases. Figure 2 shows the chromatograms of NSAID precursor ions from a milk sample spiked at $0.5 \mu \mathrm{g} \cdot \mathrm{kg}^{-1}$ (a) and at $0.1 \mu \mathrm{g} \cdot \mathrm{kg}^{-1}$ (b).

Fig. 2

LC-HRMS/MS chromatograms of precursor ions. Milk samples spiked at: (a) $0.5 \mu \mathrm{g} \cdot \mathrm{kg}^{-1}$; (b) $0.1 \mu \mathrm{g} \cdot \mathrm{kg}^{-1}$

\section{Conclusions}

The QuEChERS methodology has proven to be a reliable strategy for analyzing NSAIDs in milk samples using LC-MS/MS. The new method allows high sample throughput thanks to its simplicity and effectiveness, and is very suitable for control laboratories. The method has been validated, and its successful performance in proficiency tests demonstrates its ability to provide accurate results.

We have also investigated the use of HRMS/MS. The high accuracy, resolution, and sensitivity provided by the Q-Orbitrap instrument is especially suitable for reliable analysis at very low concentrations, such as that required for diclofenac, the MRL 
of which is established by EU legislation at $0.1 \mu \mathrm{g} \cdot \mathrm{kg}^{-1}$. Although this instrumentation still has a limited presence in food safety testing laboratories, it is useful for routine analysis and may become an excellent tool for complex samples, and an alternative to triple quadrupole mass spectrometers.

The new method is currently used in control plans implemented in the area of Catalonia, and was used to analyze more than 50 samples of milk from different animal species in 2015.

\section{Acknowledgments}

The authors thank Jojo Tibon for his contribution to the HRMS experiments. Compliance with ethical standards

Conflict of interest

The authors declare that they have no conflict of interest.

\section{Electronic supplementary material}

Below is the link to the electronic supplementary material.

\section{ESM 1}

(PDF $429 \mathrm{~kb})$

\section{References}

1.

Sweetman SC, editor. Martindale. The Complete Drug Reference. 37th ed. London: Pharmaceutical Press: Royal Pharmaceutical Society; 2011.

2.

Commission Regulation (EU) Nr. 37/2010 of 22 December 2009 on pharmacologically active substances and their classification regarding maximum residue limits in foodstuffs of animal origin. Off J Eur Union. 2009; L 15/1.

3.

CRL Guidance Paper. CRL's View on the art of Analytical Methods for National residue control Plans. 2007. Available at:http://www.bvl.bund.de/SharedDocs/Downloads/09_Untersuchungen/EURL_Em pfehlungen_Konzentrationsauswahl_Methodenvalierungen_EN.pdf;jsessionid=75F 6F1917236B2A4DBCF5145859A6C22.2_cid332?_blob=publicationFile\&v=2http: //www.bvl.bund.de/SharedDocs/Downloads/09_Untersuchungen/EURL_Empfehlun gen_Konzentrationsauswahl_Methodenvalierungen_EN.pdf?_blob=publicationFile $\& v=2$. Accessed 29 Feb 2016.

4.

Malone EM, Dowling G, Elliot CT, Kennedy DG, Regan L. Development of a rapid, multiclass method for the confirmatory analysis of anti-inflammatory drugs 
in bovine milk using liquid chromatography tandem mass spectrometry. $\mathrm{J}$ Chromatogr A. 2009;1216:8132-40.

5.

Gentili A, Caretti F, Bellante S, Mainero Rocca L, Curini R, Venditti A. Development and validation of two multiresidue liquid chromatography tandem mass spectrometry methods based on a versatile extraction procedure for isolating non-steroidal anti-inflammatory drugs from bovine milk and muscle tissue. Anal Bioanal Chem. 2012;404:1375-88.

6.

Jedziniak P, Szprengier-Juszkiewicz T, Pietruk K, Sledzinska E, Zumdzki. Determination of non-steroidal anti-inflammatory drugs and their metabolites in milk by liquid chromatography-tandem mass spectrometry. Anal Bioanal Chem. 2012;403:2955-63.

7.

Dubreil-Chéneau E, Pirotais Y, Bessiral M, Roudaut B, Verdon E. Development and validation of a confirmatory method for the determination of 12 antiinflammatory drugs in milk using liquid chromatography-tandem mass spectrometry. J Chromatogr A. 2011;1218:6292-301.

8.

Van Pamel E, Daeseleire E. A multiresidue liquid chromatographic tandem mass spectrometric method for the detection and quantitation of 15 non-steroidal antiinflammatory drugs (NSAIDs) in bovine meat and milk. Anal Bioanal Chem. 2015;407:4485-94.

9.

Peng T, Zhu AL, Zhou YN, et al. Development of a simple method for simultaneous determination of nine subclasses of non-steroidal anti-inflammatory drugs in milk and dairy products by ultra-performance liquid chromatography with tandem mass spectrometry. J Chromatogr B. 2013;933:15-23.

10.

Stolker AAM, Rutgers P, Oosterink E, et al. Comprehensive screening and quantification of veterinary drugs in milk using UPLC-ToF-MS. Anal Bioanal Chem. 2008;391:2309-22.

11.

Dowling G, Gallo P, Fabroccino S, Serpe L, Regan L. Determination of ibuprofen, ketoprofen, diclofenac, and phenylbutazone in bovine milk by gas chromatography tandem mass spectrometry. Food Addit Contam Part A. 2008;25:1497-508. 
12.

Gallo P, Fabroccino S, Vinci F, Fiori M, Danese V, Serpe L. Confirmatory identification of sixteen non-steroidal anti-inflammatory drug residues in raw milk by liquid chromatography coupled with ion trap mass spectrometry. Rapid Commun Mass Spectrom. 2008;22:841-54.

13.

Anastassiades M, Lehotay SJ, Stajnbaher D, Schenck FJ. Fast and easy multiresidue method employing acetonitrile extraction/ partitioning and dispersive solid-phase extraction for the determination of pesticide residues in procedure. J AOAC Int. 2003;86:412-43.

14.

Moragues F, Igualada C, León N. Qualitative confirmatory method for determination of Phenylbutazone and 11 prohibited NSAIDs in animal tissue and milk by UPLC-MS/MS. Prague: Recent Advances in Food Analysis; 2013.

15.

Commission Decision 2002/657/EC of 12 August 2002 implementing Council Directive 96/23/EC concerning the performance of analytical methods and the interpretation of results. Off J Eur Union. 2002; L 221/8-36.

16.

ISO 11843-2. Capability of detection. Part 2: Methodology in the linear calibration case. 2nd ed. International Organization for Standardization; 2000. 